\title{
Global dynamo models from direct numerical simulations and their mean-field counterparts
}

\begin{abstract}
M. Schrinner ${ }^{1}$
MAG (ENS/IPGP), LRA, Ecole Normale Supérieure, 24 rue Lhomond, 75252 Paris Cedex 05, France

e-mail: martin@schrinner .eu

Received 3 February 2011 / Accepted 24 June 2011

ABSTRACT

Context. The test-field method permits us to compute dynamo coefficients from global, direct numerical simulations. The subsequent use of these parameters in mean-field models enables us to compare self-consistent dynamo models with their mean-field counterparts. This has been done to date for a simulation of rotating magnetoconvection and a simple benchmark dynamo, which are both (quasi-)stationary.

Aims. It is shown that chaotically time-dependent dynamos in a low Rossby number regime can be appropriately described by corresponding mean-field results. We also identify conditions under which mean-field models disagree with direct numerical simulations. Methods. We solve the equations of magnetohydrodynamics (MHD) in a rotating, spherical shell in the Boussinesq approximation. Based on this, we compute mean-field coefficients for several models with the help of the previously developed test-field method. The parameterization of the mean electromotive force by these coefficients is tested against direct numerical simulations. In addition, we use the determined dynamo coefficients in mean-field models and compare the outcome with azimuthally averaged fields from direct numerical simulations.

Results. The azimuthally and time-averaged electromotive force in rapidly rotating dynamos is sufficiently well parameterized by the set of determined mean-field coefficients. In comparison to the previously considered (quasi-)stationary dynamo, the chaotic timedependence leads to an improved scale separation and thus to a closer agreement between direct numerical simulations and mean-field results.
\end{abstract}

Key words. methods: numerical - magnetohydrodynamics (MHD) - dynamo

\section{Introduction}

Mean-field electrodynamics provides a conceptual understanding of dynamo processes generating coherent, large-scale magnetic fields in planets, stars, and galaxies (Krause \& Rädler 1980; Moffatt 1978). However, it depends on a number of free parameters, that are only poorly known under astrophysically relevant conditions. We briefly summarize the essentials of mean-field theory to specify this point. Within a mean-field approach, the velocity and the magnetic field are usually divided into largescale mean fields, $\overline{\boldsymbol{V}}, \overline{\boldsymbol{B}}$, and residual parts, $\boldsymbol{v}, \boldsymbol{b}$, that vary on much shorter length or timescales,

$\boldsymbol{V}=\overline{\boldsymbol{V}}+\boldsymbol{v}, \quad \boldsymbol{B}=\overline{\boldsymbol{B}}+\boldsymbol{b}$

The evolution of the mean field is then governed by

$\frac{\partial \overline{\boldsymbol{B}}}{\partial t}=\nabla \times(\mathcal{E}+\overline{\boldsymbol{V}} \times \overline{\boldsymbol{B}}-\eta \nabla \times \overline{\boldsymbol{B}})$.

In the above equation, the mean electromotive force is defined as $\mathcal{E}=\overline{\boldsymbol{v} \times \boldsymbol{b}}$ and $\eta$ is the magnetic diffusivity. Equation (2) is coupled with an equation for the residual field

$\frac{\partial \boldsymbol{b}}{\partial t}=\nabla \times(\boldsymbol{G}+\overline{\boldsymbol{V}} \times \boldsymbol{b}+\boldsymbol{v} \times \overline{\boldsymbol{B}}-\eta \nabla \times \boldsymbol{b})$,

in which $\boldsymbol{G}$ is defined as $\boldsymbol{G}=\boldsymbol{v} \times \boldsymbol{b}-\overline{\boldsymbol{v} \times \boldsymbol{b}}$. The above system of equations is equivalent to the usual induction equation and no approximation has been applied so far. An important simplification of the mean-field approach consists of considering Eq. (2) alone, which requires us to express $\mathcal{E}$ as a linear functional of $\boldsymbol{v}, \overline{\boldsymbol{V}}$, and $\overline{\boldsymbol{B}}$. This is justified by Eq. (3), and we may write

$\mathcal{E}_{i}=\mathcal{E}_{i}^{(0)}+\iint \mathrm{K}_{i j}\left(\boldsymbol{x}, \boldsymbol{x}^{\prime}, t, t^{\prime}\right) \overline{\boldsymbol{B}}_{j}\left(\boldsymbol{x}^{\prime}, t^{\prime}\right) \mathrm{d}^{3} x^{\prime} \mathrm{d} t^{\prime}$,

in which $\mathrm{K}$ denotes some integral kernel and we refer for the moment to cartesian coordinates. The term $\mathcal{E}^{(0)}$ in Eq. (4) accounts for small-scale dynamo action and is not considered here but discussed later. In addition, we assume that $\mathcal{E}$ depends only instantaneously and nearly locally on $\overline{\boldsymbol{B}}$. This is crucial and is referred to as the assumption of scale separation in the following. Therefore, $\bar{B}$ in Eq. (4) may be replaced by its rapidly converging Taylor series expansion at $\boldsymbol{x}$,

$\overline{\boldsymbol{B}}_{j}\left(\boldsymbol{x}^{\prime}, t\right)=\overline{\boldsymbol{B}}_{j}(\boldsymbol{x}, t)+\left(x_{k}^{\prime}-x_{k}\right) \frac{\partial \overline{\boldsymbol{B}}_{j}(\boldsymbol{x}, t)}{\partial x_{k}}+\cdots$,

and taken out of the integral

$\mathcal{E}_{i}=\mathrm{a}_{i j} \overline{\boldsymbol{B}}_{j}+\mathrm{b}_{i j k} \frac{\partial \overline{\boldsymbol{B}}_{j}}{\partial x_{k}}+\cdots$,

where

$\mathrm{a}_{i j}=\iint \mathrm{K}_{i j}\left(\boldsymbol{x}, \boldsymbol{x}^{\prime}, t, t^{\prime}\right) \mathrm{d}^{3} x^{\prime} \mathrm{d} t^{\prime}$,

$\mathrm{b}_{i j k}=\iint \mathrm{K}_{i j}\left(\boldsymbol{x}, \boldsymbol{x}^{\prime}, t, t^{\prime}\right)\left(x_{k}^{\prime}-x_{k}\right) \mathrm{d}^{3} x^{\prime} \mathrm{d} t^{\prime}$ 
The dynamo coefficients $a$ and $b$ defined in Eqs. (7) and (8) together with the expansion in Eq. (6) may finally be used to solve Eq. (2). Moreover, $a$ and $b$ are directly linked to dynamo processes, i.e. to the generation, advection, and diffusion of the mean magnetic field, thus provide physical concepts to explain dynamo action (e.g. Rädler 1995). Unfortunately, they are not known in general and previous work often relies on arbitrary assumptions about them.

The test-field method by Schrinner et al. $(2005,2007)$ permits us to compute $a$ and $b$ from direct numerical simulations. It was first applied to a simulation of rotating magnetoconvection (Olson et al. 1999) and a simple geodynamo simulation (Christensen et al. 2001), which are both stationary except for an azimuthal drift. In the past three years, the test-field method was intensively used to compute mean-field coefficients for box simulations (e.g. Sur et al. 2008; Gressel et al. 2008; Brandenburg 2009; Käpylä et al. 2009; Rädler \& Brandenburg 2009), which are not the focus of this paper. Although the test-field method proved to be reliable, the parameterization of the electromotive force for the steady geodynamo model was unsatisfactory. The expansion in Eq. (6) does not converge for this steady example because of a non-sufficient scale separation (Schrinner et al. 2007). In this paper, we revisit the problem for chaotically time-dependent dynamos. We test azimuthally and timeaveraged electromotive-force vectors against their parameterization based on corresponding dynamo coefficients and compare azimuthally and time-averaged fields from direct numerical simulations with mean-field results.

\section{Dynamo simulations}

We solve the equations of magnetohydrodynamics (MHD) in the Boussinesq approximation for a conducting fluid in a rotating spherical shell as given by Olson et al. (1999),

$$
\begin{gathered}
E\left(\frac{\partial \boldsymbol{V}}{\partial t}+\boldsymbol{V} \cdot \nabla \boldsymbol{V}-\nabla^{2} \boldsymbol{V}\right)+2 \boldsymbol{z} \times \boldsymbol{V}+\nabla P= \\
R a \frac{\boldsymbol{r}}{r_{o}} T+\frac{1}{P m}(\nabla \times \boldsymbol{B}) \times \boldsymbol{B} \\
\frac{\partial \boldsymbol{B}}{\partial t}=\nabla \times(\boldsymbol{V} \times \boldsymbol{B})+\frac{1}{P m} \nabla^{2} \boldsymbol{B} \\
\frac{\partial T}{\partial t}+\boldsymbol{V} \cdot \nabla T=\frac{1}{P r} \nabla^{2} T .
\end{gathered}
$$

The coupled differential equations for the the velocity $\boldsymbol{V}$, the magnetic field $\boldsymbol{B}$, and the temperature $T$ are governed by four parameters. These are the Ekman number $E=v / \Omega L^{2}$, the (modified) Rayleigh number $R a=\alpha_{\mathrm{T}} g_{0} \Delta T L / v \Omega$, the Prandtl number $\operatorname{Pr}=v / \kappa$, and the magnetic Prandtl number $P m=v / \eta$. In these expressions, $v$ denotes the kinematic viscosity, $\Omega$ the rotation rate, $L$ the shell width, $\alpha_{\mathrm{T}}$ the thermal expansion coefficient, $g_{0}$ is the gravitational acceleration at the outer boundary, $\Delta T$ stands for the temperature difference between the spherical boundaries, $\kappa$ is the thermal and $\eta=1 / \mu \sigma$ the magnetic diffusivity with the magnetic permeability $\mu$, and the electrical conductivity $\sigma$. In addition to these input parameters, we introduce the magnetic Reynolds number $R m=V_{\text {rms }} L / \eta$, and the local Rossby number $R o_{l}=V_{\text {rms }} /(\Omega L) \cdot(\bar{l} / \pi)$ as important output parameters. For the latter parameter definitions, $V_{\text {rms }}$ denotes the rms-velocity and $\pi / \bar{l}$ is the mean half-wavelength of the flow derived from the kinetic energy spectrum (Christensen \& Aubert 2006).

No-slip mechanical boundary conditions were chosen for all simulations presented here and the magnetic field continues as a potential field outside the fluid shell. Convection is driven by an imposed temperature difference between the inner and the outer shell shell-boundary.

\section{Computation of dynamo coefficients}

To determine the dynamo coefficients $a$ and $b$, we apply the testfield method explained in full detail in Schrinner et al. (2007). The principal idea of this approach is to measure the mean electromotive force generated by the interaction of the flow with an arbitrarily imposed test field, $\bar{B}_{\mathrm{T}}$. The imposed test field appears as an inhomogeneity in Eq. (3),

$$
\frac{\partial \boldsymbol{b}}{\partial t}-\nabla \times(\boldsymbol{G}+\overline{\boldsymbol{V}} \times \boldsymbol{b}-\eta \nabla \times \boldsymbol{b})=\nabla \times \boldsymbol{v} \times \overline{\boldsymbol{B}}_{\mathrm{T}},
$$

which is solved simultaneously with Eqs. (9)-(11). The electromotive force due to a given $\overline{\boldsymbol{B}}_{\mathrm{T}}$ may then be computed as $\mathcal{E}_{\mathrm{T}}=\overline{\boldsymbol{v} \times \boldsymbol{b}}$ with $\boldsymbol{b}$ resulting from Eq. (12). Finally, $\mathcal{E}_{\mathrm{T}}$ is used to solve Eq. (6) for the dynamo coefficients. To close the resulting system of linear equations and determine all components of a and $b$, the numerical experiment in Eq. (12) must be repeated several times with different fields $\overline{\boldsymbol{B}}_{\mathrm{T}}$.

So far, averaged quantities labelled by an overbar are axisymmetric fields, whereas $\boldsymbol{v}$ and $\boldsymbol{b}$ in (12) are non-axisymmetric residuals. For a stochastically stationary but nevertheless chaotically time-dependent flow, $\mathcal{E}$ and the mean-field coefficients vary in time. Thus, we introduce an additional time-averaging indicated by brackets, $\langle\cdots\rangle$, and write

$\mathcal{E} \approx \mathrm{a}\langle\overline{\boldsymbol{B}}\rangle+\mathrm{b}\langle\nabla \overline{\boldsymbol{B}}\rangle+\cdots$

instead of Eq. (6). In the above equation, $\mathcal{E}$ has been timeaveraged,

$\mathcal{E}=\langle\overline{\boldsymbol{v} \times \boldsymbol{b}}\rangle$,

and $a$ and $b$ also denote time-averaged tensors. Furthermore, we interpret averages in Eq. (2) as combined azimuthal and time averages. Neither is formally correct. However, the approximations applied can be justified, if

i) temporal fluctuations of the axisymmetric velocity and magnetic field are comparatively small, and

ii) time averages of the non-axisymmetric residuals of the velocity and the magnetic field are negligible.

The above assumptions imply that azimuthal and time averaging act in a similar way and reinforce each other. As a consequence, mean-field coefficients are derived in this context from time averages of vectors $\mathcal{E}_{\mathrm{T}}$ resulting from Eq. (12). We emphasise that assumptions i) and ii) are not justified a priori but have to be tested in numerical simulations.

\section{Comparison of mean-field results with direct numerical simulations}

Mean-field coefficients determined from direct numerical simulations may be used in mean-field models. Subsequently, azimuthally and time-averaged magnetic fields resulting from three-dimensional, self-consistent models may be compared with their mean-field counterparts. Mean-field calculations presented here are based on Eq. (2) written as an eigenvalue problem

$\sigma \overline{\boldsymbol{B}}=\nabla \times \mathrm{D} \overline{\boldsymbol{B}}$ 
Table 1. Overview of the models considered, ordered with respect to their local Rossby number.

\begin{tabular}{lcccccccc}
\hline \hline Model & $E$ & $R a$ & $P m$ & Mean 1 & $R o_{l}$ & $R m$ & $\Delta \mathcal{E}$ & $\lambda /\left(\eta / L^{2}\right)$ \\
\hline 1 & $1 \times 10^{-3}$ & 100 & 5 & 5 & 0.013 & 39 & 4.6 & 4.18 \\
2 & $1 \times 10^{-4}$ & 334 & 3 & 11 & 0.014 & 123 & 1.6 & -7.05 \\
3 & $1 \times 10^{-4}$ & 334 & 2 & 11 & 0.015 & 86 & 1.4 & -3.87 \\
4 & $3 \times 10^{-4}$ & 195 & 3 & 9 & 0.019 & 67 & 1.6 & 2.22 \\
5 & $3 \times 10^{-4}$ & 243 & 2 & 9 & 0.024 & 56 & 1.2 & 0.66 \\
6 & $3 \times 10^{-4}$ & 285 & 2 & 9 & 0.026 & 61 & 1.3 & 0.05 \\
7 & $3 \times 10^{-4}$ & 375 & 1.5 & 11 & 0.042 & 60 & 1.1 & 0.78 \\
\hline
\end{tabular}

in which the linear operator $D$ is defined as

$\mathrm{D} \overline{\boldsymbol{B}}=\overline{\boldsymbol{V}} \times \overline{\boldsymbol{B}}+\mathrm{a} \overline{\boldsymbol{B}}+\mathrm{b} \nabla \overline{\boldsymbol{B}}-1 / P m \nabla \times \overline{\boldsymbol{B}}$,

and $\mathrm{a}$ and $\mathrm{b}$ are time-averaged dynamo coefficients as described above. The eigenvalues $\sigma$ lead to a time evolution of each mode that is proportional to $\exp (\sigma t)$. For more details about the eigenvalue calculation, we refer to Schrinner et al. (2010b).

In addition, the parameterization of the electromotive force by dynamo coefficients, i.e. Eq. (6), may be tested against the mean electromotive force in direct numerical simulations. Again by virtue of Eqs. (13), (14), we compare $\mathcal{E}$ as given in Eq. (14) and derived directly from numerical models, with

$\mathcal{E}^{(1)}=\mathrm{a}\langle\overline{\boldsymbol{B}}\rangle+\mathrm{b}\langle\nabla \overline{\boldsymbol{B}}\rangle$,

in which $\langle\overline{\boldsymbol{B}}\rangle$ and $\langle\nabla \overline{\boldsymbol{B}}\rangle$ are also obtained from direct numerical simulations. Furthermore, we follow Schrinner et al. (2007) and introduce

$\Delta \mathcal{E}=\frac{\int_{V}\left|\mathcal{E}-\mathcal{E}^{(1)}\right| \mathrm{d} V}{\int_{V}|\mathcal{E}| \mathrm{d} V}$

in order to quantify errors in the parameterization of $\mathcal{E}$.

\section{Results}

Besides the (quasi-)steady benchmark dynamo (model 1), we considered six chaotically time-dependent dynamos. The models are defined by four control parameters; $E, R a$, and $P m$ were varied (see Table 1), whereas the Prandtl number was kept fixed and set to 1 for all simulations. The local Rossby number is always lower than 0.12 for the models considered here. They therefore belong to the regime of kinematically stable dynamos (Schrinner et al. 2010a). To simplify the time averaging, models with low and fairly moderate $R m$ were chosen. The resulting velocity fields are almost symmetric with respect to the equatorial plane, except for model 7 , and convection occurs mainly outside the inner core tangent cylinder. Figure 1 shows the volumeaveraged kinetic energy density for model 3 and illustrates the irregular time-dependence of these models.

The parameterization of the mean electromotive force given by Eq. (17) was found to be more suitable for the chaotically time-dependent dynamos than the benchmark dynamo; $\Delta \mathcal{E}$ decreases by more than a factor of 4 from model 1 to model 7 . The considerable decline in $\Delta \mathcal{E}$ from the steady to the timedependent models is most clearly visible in Fig. 2. It shows $\Delta \mathcal{E}$ for different models versus their magnetic Reynolds number. Apart from the aforementioned difference between the timedependent and the stationary models, a dependence of $\Delta \mathcal{E}$ on $R m$ cannot be inferred.

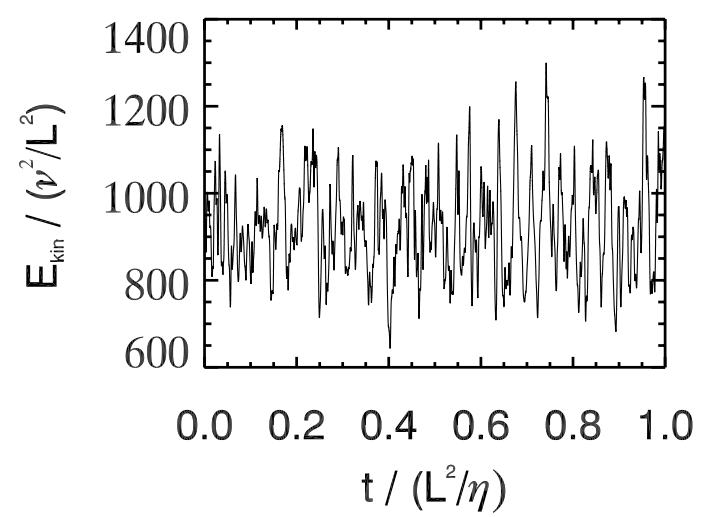

Fig. 1. Volume-averaged kinetic energy density versus magnetic diffusion time for model 3 .

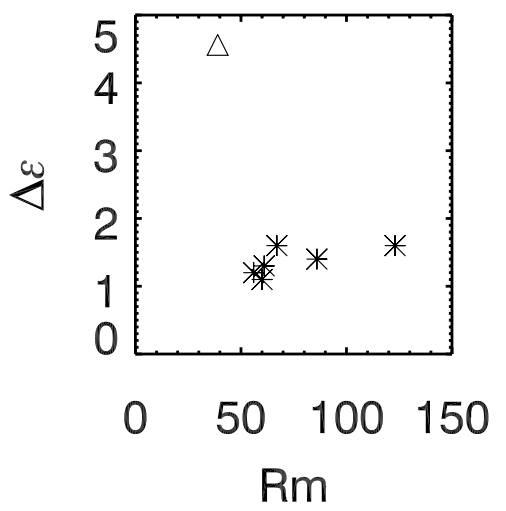

Fig. 2. $\Delta \varepsilon$ versus the magnetic Reynolds number for the (quasi)stationary benchmark dynamo (triangle) and the six chaotically timedependent dynamos considered.

In addition, the growth rate of the leading eigenmode of $D$, $\lambda=\mathfrak{R}(\sigma)$, may be taken as a measure for the accuracy of the mean-field description. Ideally, it is 0 , whereas all overtones are highly diffusive (Schrinner et al. 2010a, 2011b). For numerical simulations, however, it is impossible to hit the critical point exactly. The growth rates of the fundamental mode for all models are given in Table 1 in units of $\eta / L^{2}$. We note that the turbulent diffusivity largely exceeds the molecular one. For model 2 , for instance, we find values of $\beta$-components up to $63 \eta$. Thus, for model $2,1 / \lambda \approx 1 / 7 L^{2} / \eta$ is much larger than one effective decay time and the fundamental mode is already near its critical state. With decreasing $\Delta \mathcal{E}$, the growth rates become even smaller and the values closest to zero have been obtained for models 5-7.

Model 7 provided the closest fitting and enabled $\Delta \mathcal{E}=1.1$ to be achieved. This is still clearly larger than for the simulation of rotating magnetoconvection for which $\Delta \mathcal{E}=0.28$ was found by Schrinner et al. (2007). Figure 3 compares $\mathcal{E}$ to $\mathcal{E}^{(1)}$. Differences are visible in all components, but also principal similarities.

Figure 4 compares the azimuthally and time-averaged magnetic field resulting from direct numerical simulations with the fundamental eigenmode of $\mathrm{D}$ for model 7. Apart from small differences, direct numerical simulations and mean-field calculations agree very well for this example.

\section{Discussion}

We do not derive mean-field coefficients for time-averaged mean fields in a formal sense. The comparisons in Figs. 3 and 4 are motivated by assumptions i) and ii) in Sect. 3, instead. They 


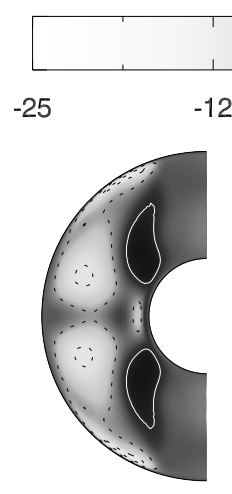

$-12$

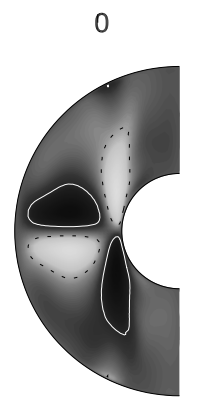

12 25
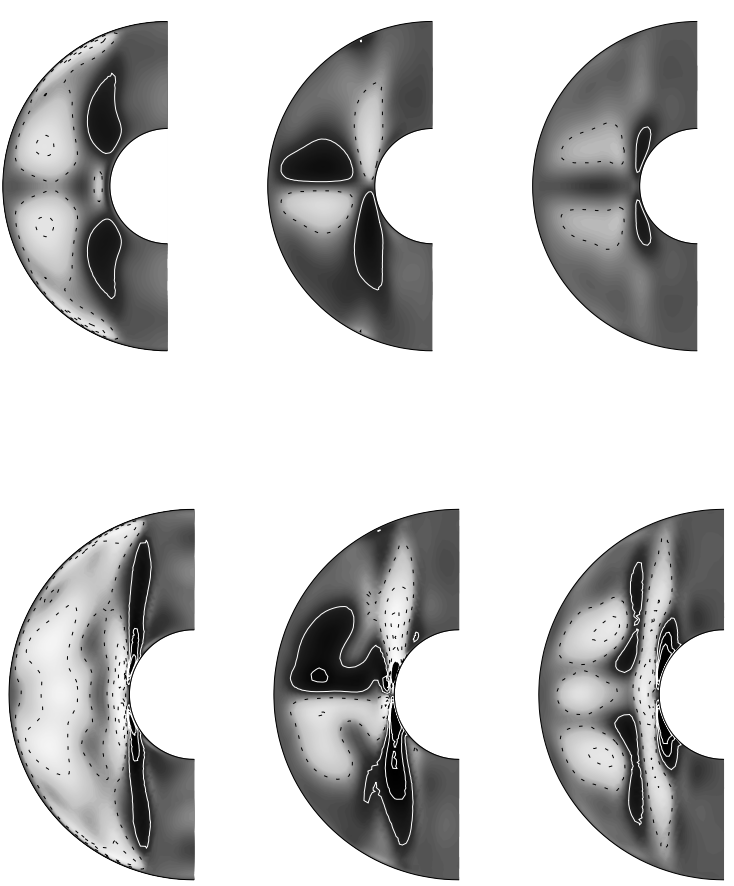

Fig. 3. Contour plots of $\mathcal{E}_{r}, \mathcal{E}_{\theta}$, and $\mathcal{E}_{\phi}$ (top line, from left to right) and $\mathcal{E}_{r}^{(1)}, \mathcal{E}_{\theta}^{(1)}$, and $\mathcal{E}_{\phi}^{(1)}$ (bottom line, from left to right) for model 7 in units of $(v / L)(\varrho \mu \eta \Omega)^{1 / 2}$. In these units, contour lines correspond to $\pm 3, \pm 9, \pm 15$.

are reasonably well fulfilled for the simulations considered here. For model 7, $v$ time-averaged over 30 magnetic diffusion times declines sharply to $0.1 \%$ of $\boldsymbol{V}$. A similar decrease has been found for $\boldsymbol{b}$. Moreover, the axisymmetric flow and the axisymmetric magnetic field show comparatively little time variation. The time-dependent components of $\overline{\boldsymbol{V}}$ and $\overline{\boldsymbol{B}}$ are of the order of $8 \%(37 \%)$ and $17 \%(23 \%)$ of the total (axisymmetric) flow and the total (axisymmetric) magnetic field, respectively. In other words, time-averaging results in axisymmetric fields and azimuthal averaging somewhat reduces the time variability. Combining both leads to an extended averaging and thus to an improved scale separation. This finding is also supported by Figs. 5 and 6. Figure 5 shows a noticeable decrease of $\Delta \mathcal{E}$ for model 5 , if the time-averaging period $\tau$ is increased. $\Delta \mathcal{E}$ drops rapidly until $\tau \approx 2 L^{2} / \eta$; an extension of the time-averaging interval over more than 2 magnetic diffusion times improves the scale separation only slightly. Moreover, Fig. 6 compares the residual with the mean magnetic field for model 1 (upper panel) and model 7 (lower panel). In contrast to model 1, the time-dependent residual and the time-averaged mean magnetic field of model 7 vary on fairly different length-scales. Thus, a clearer scale separation for the time-dependent models seems to be plausible. Consequently, $\Delta \mathcal{E}$ drops drastically from model 1 to model 2 . However, there are still noticeable differences between $\mathcal{E}$ and $\mathcal{E}^{(\mathbf{1})}$ in Fig. 3 for model 7. On the basis of this comparison alone, it would be difficult to decide, whether the parameterization of $\mathcal{E}$ is already satisfactory. Additional support for its reliability comes from the good agreement between the results of direct numerical simulations and mean-field modeling in Fig. 4. Contour plots of all three components of the leading eigenmode of $D$ agree well with corresponding results from
$B_{r}$

$\mathrm{B}_{\vartheta}$
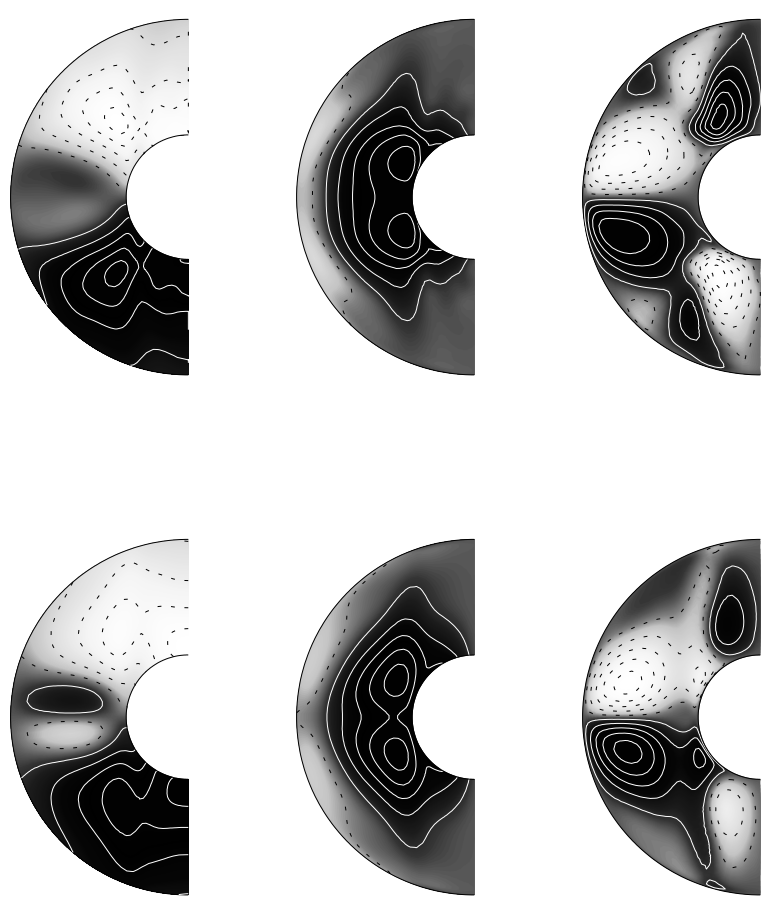

Fig. 4. Comparison between direct numerical simulations and meanfield calculations for model 7. Bottom line: azimuthally and timeaveraged magnetic field obtained by solving (9)-(11). Top line: fundamental eigenmode resulting from (15). Each component has been normalised with respect to its absolute maximum. Therefore, the greyscale coding varies from -1 , white, to +1 , black, and the contour lines correspond to $\pm 0.1, \pm 0.3, \pm 0.5 \pm 0.7, \pm 0.9$.

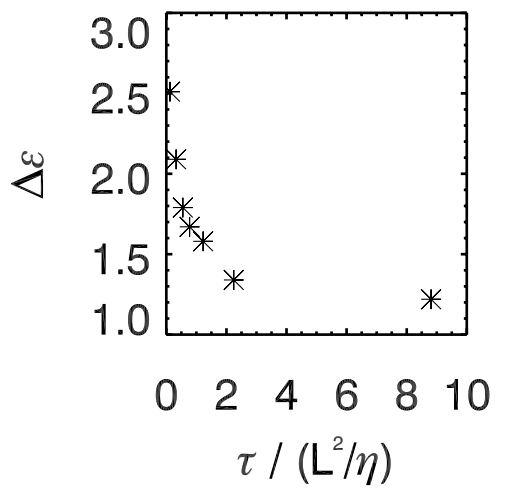

Fig. 5. $\Delta \mathcal{E}$ for model 5 varying with increasing time-averaging period $\tau$.

direct numerical simulations. Moreover, the growth rates of the leading eigenmodes are close to zero, as expected for stochastically stationary dynamos in the kinematically stable regime.

Dynamo action in stars and planetary cores most probably takes place on a wide range of length and time scales. Owing to computational limitations, numerical simulations do not cover the whole range of scales possibly involved. Global dynamo simulations focus only on large scales, and we typically do not find small-scale dynamo action as reported for box simulations (e.g. Vögler \& Schüssler 2007). Therefore, the component of the electromotive force independent of the mean magnetic field, i.e. $\mathcal{E}^{(0)}$ in (4), is zero for most global dynamo simulations. The 

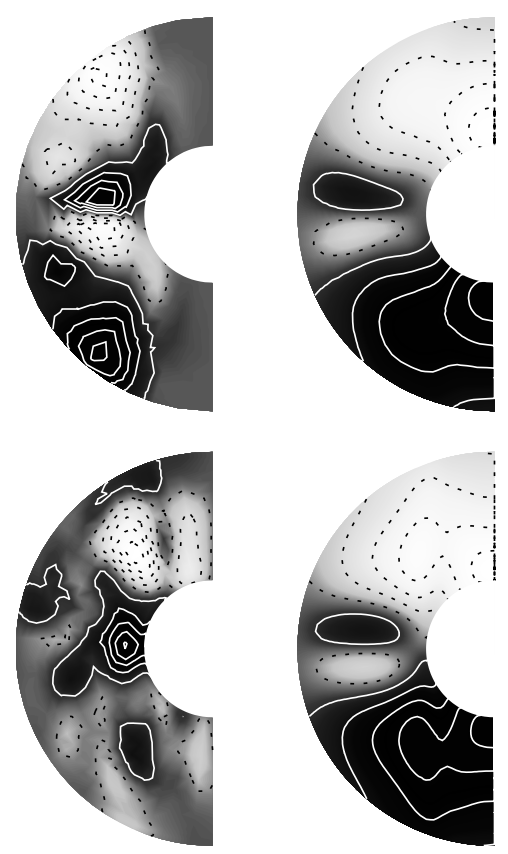

Fig. 6. Upper panel: non-axisymmetric radial magnetic field at arbitrary longitude (left) and azimuthally-averaged radial magnetic field (right) for model 1. Lower panel: non-axisymmetric and time-dependent radial magnetic field at arbitrary longitude and time (left) and azimuthally and time-averaged radial magnetic field (right) for model 7. The style of the contour plots is the same as in Fig. 4.

possibility of a contribution to $\mathcal{E}$ from small-scale dynamo action has been intensively discussed in the literature (e.g. Rädler 1976; Rädler 2000; Rädler \& Rheinhardt 2007). It has been used to argue against the applicability of mean-field theory (Cattaneo $\&$ Hughes 2009). However, the conceptual difficulties that might result from simultaneous small- and large-scale dynamo action are at present irrelevant to the approach followed here.

The problem of scale separation has been addressed in this study only on spatial scales. This seems to be appropriate because we intend to describe stochastically stationary mean-fields with zero growth rate resulting from self-consistent dynamo simulations. More generally, time derivatives of the mean field in the expansion of $\mathcal{E}$ have to be taken into account. As for nonlocal effects (Brandenburg et al. 2008), memory effects of the flow may also influence the parameterization of the electromotive force (Hubbard \& Brandenburg 2009; Hughes \& Proctor 2010).

Mean-field theory as presented here is intrinsically a kinematic approach. In general, an eigenvalue calculation based on Eq. (15) will lead to growing modes, even if the mean-field coefficients are derived from a saturated velocity field (Tilgner \& Brandenburg 2008; Cattaneo \& Tobias 2009). In this situation, mean-field models might not agree with direct numerical simulations, unless a more self-consistent extension of the theoretical framework and the test-field method is applied (Courvoisier et al. 2010; Rheinhardt \& Brandenburg 2010). However, the class of chaotically time-dependent dynamos we considered is kinematically stable. Schrinner et al. (2010a) identified a regime of rapidly rotating dynamos that are dominated by only one dipolar mode at marginal stability, whereas all overtones are highly diffusive. A saturated velocity field from this class of dynamos does not lead to exponential growth of the magnetic field in a corresponding kinematic calculation. Consequently, the meanfield approach based on Eq. (15) is applicable to this dynamo regime, as confirmed once more in this study. In particular for models beyond this regime, the reliability of the mean-field approach presented here is not guaranteed and has to be demonstrated to be plausible by a comparison with direct numerical simulations (e.g. Schrinner et al. 2011a).

\section{Summary}

The mean-field description of a (quasi-)stationary dynamo has been affected by a poor scale-separation, hence from an insufficiently accurate parameterization of the electromotive force (Schrinner et al. 2007). We have demonstrated that the chaotically time-dependent dynamos considered here can provide improvements in both cases, if a combined azimuthal and time average is applied. The more accurate parameterization of $\mathcal{E}$ leads to close agreement between mean-field models and direct numerical simulations: field topologies and growth rates resulting from both approaches have indeed be shown to be very similar.

In conclusion, the test-field method for determining meanfield coefficients from direct numerical simulations has also been found to be reliable for chaotically time-dependent dynamos. Mean-field theory may serve as a powerful tool to analyse the dynamo processes in global models resulting from direct numerical simulations.

Acknowledgements. M.S. is grateful for financial support from the ANR Magnet project. The computations were carried out at the French national computing center CINES

\section{References}

Brandenburg, A. 2009, Space Sci. Rev., 144, 87

Brandenburg, A., Rädler, K., \& Schrinner, M. 2008, A\&A, 482, 739

Cattaneo, F., \& Hughes, D. W. 2009, MNRAS, 395, L48

Cattaneo, F., \& Tobias, S. M. 2009, J. Fluid Mech., 621, 205

Christensen, U. R., \& Aubert, J. 2006, Geophy. J. Int., 166, 97

Christensen, U. R., Aubert, J., Cardin, P., et al. 2001, Phys. Earth Planet. Inter., 128,25

Courvoisier, A., Hughes, D. W., \& Proctor, M. R. E. 2010, Proc. Roy. Soc. Lond., 466,583

Gressel, O., Ziegler, U., Elstner, D., \& Rüdiger, G. 2008, Astron. Nachr., 329, 619

Hubbard, A., \& Brandenburg, A. 2009, ApJ, 706, 712

Hughes, D. W., \& Proctor, M. R. E. 2010, Phys. Rev. Lett., 104, 024503

Käpylä, P. J., Korpi, M. J., \& Brandenburg, A. 2009, A\&A, 500, 633

Krause, F., \& Rädler, K. 1980, Mean-field magnetohydrodynamics and dynamo theory (Oxford: Pergamon Press)

Moffatt, H. K. 1978, Magnetic field generation in electrically conducting fluids (Cambridge: Cambridge University Press)

Olson, P., Christensen, U. R., \& Glatzmaier, G. A. 1999, J. Geophys. Res., 104, 10383

Rädler, K.-H. 1976, in Basic Mechanisms of Solar Activity, ed. V. Bumba, \& J. Kleczek, IAU Symp., 71, 323

Rädler, K.-H. 1995, in Rev. Mod. Astron., ed. G. Klare, 8, 295

Rädler, K.-H. 2000, in From the Sun to the Great Attractor, ed. D. Page, \& J. G. Hirsch (Berlin: Springer Verlag), Lecture Notes in Physics, 556, 101

Rädler, K.-H., \& Brandenburg, A. 2009, MNRAS, 393, 113

Rädler, K.-H., \& Rheinhardt, M. 2007, Geophys. Astrophys. Fluid Dyn., 101, 117

Rheinhardt, M., \& Brandenburg, A. 2010, A\&A, 520, A28

Schrinner, M., Rädler, K.-H., Schmitt, D., Rheinhardt, M., \& Christensen, U. R. 2005, Astron. Nachr., 326, 245

Schrinner, M., Rädler, K.-H., Schmitt, D., Rheinhardt, M., \& Christensen, U. R. 2007, Geophys. Astrophys. Fluid Dyn., 101, 81

Schrinner, M., Schmitt, D., Cameron, R., \& Hoyng, P. 2010a, Geophys. J. Int., 182,675

Schrinner, M., Schmitt, D., Jiang, J., \& Hoyng, P. 2010b, A\&A, 519, A80

Schrinner, M., Petitdemange, L., \& Dormy, E. 2011a, A\&A, 530, A140

Schrinner, M., Schmitt, D., \& Hoyng, P. 2011b [arXiv: 1101.3022]

Sur, S., Brandenburg, A., \& Subramanian, K. 2008, MNRAS, 385, L15

Tilgner, A., \& Brandenburg, A. 2008, MNRAS, 391, 1477

Vögler, A., \& Schüssler, M. 2007, A\&A, 465, L43 